\title{
HACIA UN ASISTENTE ROBÓTICO QUIRÚRGICO COGNITIVO
}

\author{
I.Rivas-Blanco, C. López-Casado, E. Bauzano, V.F. Muñoz \\ Departamento de Ingeniería de Sistemas y Automática \\ Universidad de Málaga, irivas@uma.es
}

\begin{abstract}
Resumen
Este trabajo presenta una arquitectura cognitiva para un asistente robótico camarógrafo, cuyo objetivo consiste en ofrecer el mejor punto de vista durante la intervención quirúrgica de forma autónoma. Este sistema está formado por un minirobot cámara que se encuentra en el interior de la cavidad abdominal y un brazo robótico externo que maneja dicha cámara mediante una interacción magnética. La arquitectura cognitiva dota al asistente robótico con una memoria a largo plazo, que almacena el conocimiento quirúrgico, los comportamientos de la cámara y los mecanismos de aprendizaje, y una memoria de corto plazo que reconoce el estado actual de la tarea y lanza el comportamiento correspondiente de la cámara. Para obtener el punto de vista más apropiado de la cámara, cada estado de la tarea se caracteriza por un Foco de Atención (FA), definido por un objeto, la posición de ese objeto en la imagen y un factor de zoom. La arquitectura también incluye un mecanismo de aprendizaje que tiene en cuenta las preferencias particulares de los cirujanos durante la tarea quirúrgica y se ha validado con un conjunto de experimentos in-vitro.
\end{abstract}

Palabras Clave: Robótica quirúrgica, arquitectura cognitiva, mini robots.

\section{INTRODUCCIÓN}

En los últimos años han aparecido nuevos procedimientos de Cirugía Mínimamente Invasiva con el objetivo de reducir el número de incisiones en el paciente. En este campo, los robots asistentes quirúrgicos han encontrado una amplia gama de aplicaciones, desde robots que aumentan las capacidades del cirujano (robots extensores) hasta sistemas robóticos auxiliares que colaboran con éste durante la operación [1]. En el caso de los robots extensores, cuyo máximo referente es el sistema robótico daVinci [2], aumentan las capacidades del cirujano, así como su habilidad y destreza pero requieren entrenamiento por parte de los usuarios para manejarlos durante la operación. Por otro lado, los sistemas robóticos auxiliares son sistemas que trabajan codo con codo con el cirujano, sosteniendo la cámara laparoscópica o manejando herramientas auxiliares. Tradicionalmente, estos dispositivos son controlados directamente a través de interfaces, como head-trackers [3], eye-trackers [4]-[5] o comandos de voz [6]. Sin embargo, el uso de estos dispositivos distrae al cirujano durante la operación y es en este campo donde queda mucho por avanzar para conseguir la similitud entre un asistente humano y un robot.

Un asistente robótico ideal debe combinar tanto las capacidades humanas como las del robot bajo el concepto de co-worker [7], de manera que el robot puede colaborar de forma natural y autónoma con el cirujano. Para ello, el robot co-worker requiere de sistemas de percepción y actuación, así como de mecanismos de aprendizaje que le permitan interactuar y adaptarse a su entorno. Así, una arquitectura cognitiva con estas características puede proveer al asistente robótico con la capacidad de colaborar autónomamente con los cirujanos durante las tareas quirúrgicas, ya sea desarrollando acciones básicas pre-programadas o controlando el endoscopio. Otro requisito imprescindible en este tipo de sistema co-worker es que emule la comunicación humana, mediante la interpretación de gestos o de voz, además de dotar al robot de conocimiento quirúrgico para que identifique el estado actual de la intervención o la tarea. De esta forma, Jacob [8] desarrolló un robot que asiste al cirujano facilitándole instrumentos durante la operación y Padoy [9] y Bauzano [10] proponen sistemas colaborativos para la sutura que reconoce las acciones desarrolladas por el cirujano y actúan en consecuencia con los movimientos apropiados de asistencia. Por ejemplo, Ko [11] utiliza el análisis del flujo quirúrgico para automatizar el movimiento del endoscopio dependiendo de la herramienta en uso y Weede [12] mejora la capacidad de decisión del robot sobre el campo ideal de visión mediante la predicción a largo plazo de los movimientos de los instrumentos. Aunque estos dos últimos trabajos presentan soluciones cognitivas para el posicionamiento del endoscopio, ninguno incluye algoritmos de aprendizaje on-line para optimizar el comportamiento del robot asistente. 
En este trabajo se propone una arquitectura cognitiva para el asistente robótico camarógrafo que pretende proveer el punto de vista más apropiado de la escena en cada estado de la tarea quirúrgica. A diferencia de los trabajos previos, esta arquitectura incluye un mecanismo de aprendizaje que mejora el comportamiento del robot. De este modo, en primer lugar la sección 2 expone el sistema robótico que maneja la cámara durante las tareas laparoscópicas. A continuación, en la sección 3 se describe en detalle la arquitectura cognitiva con la memoria a largo plazo que almacena las diferentes clases de conocimiento y la memoria a corto plazo que es el motor de inferencia del sistema. Los resultados experimentales que validan la metodología se presentan en la sección 4 y las conclusiones aparecen en la sección 5.

\section{SISTEMA ROBÓTICO}

El asistente robótico está formado por un mini-robot cámara manejado por un brazo robótico externo. El mini-robot cámara, que está provisto con un conjunto de imanes de sujeción, se inserta en el interior de la cavidad abdominal a través de un puerto único de entrada (compartido con las herramientas quirúrgicas), y se adhiere al interior de la pared abdominal mediante interacción magnética. Para conseguir este propósito, el agarre magnético se fija en el efector final del brazo robótico externo. De esta forma, la colocación más apropiada de la cámara durante la operación se consigue desplazando el agarre magnético por la pared abdominal. Algunas ventajas que presenta este sistema frente a las técnicas utilizadas en la laparoscopia convencional se aprecian en que el movimiento de la cámara no está limitado por el puerto de entrada y en que se elimina la incisión para el endoscopio. Además, hay que mencionar que el guiado automático de la cámara se consigue con movimientos automatizados del brazo robótico externo que dependen de las maniobras quirúrgicas que esté ejecutando el cirujano. La identificación de estas maniobras se realiza gracias al seguimiento de la navegación de las herramientas quirúrgicas que maneja el cirujano mediante el uso de marcadores pasivos que registra un sensor de localización 3D. En la Figura 1 se muestra una visión completa del asistente robótico descrito, donde aparecen cada uno de los componentes que se han mencionado. Además, en la esquina inferior izquierda se presenta el prototipo del mini-robot cámara que se ha desarrollado. Este diseño tiene un tamaño de 30 × 22 x 90 mm, cuenta con: una cámara de alta definición (Logitech HD Webcam C310), catorce LEDS en la parte superior que iluminan el área de interés durante la operación y dos imanes permanentes en la zona posterior para su fijación en la pared abdominal.

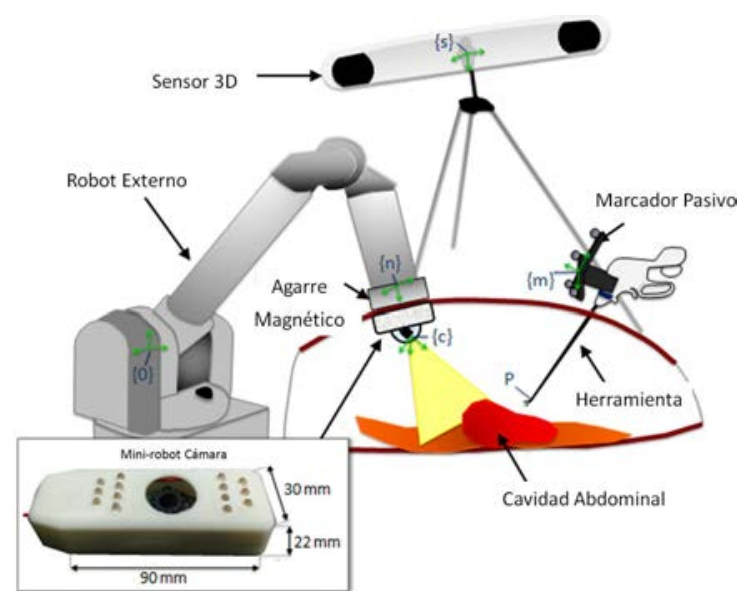

Figura 1: Asistente robótico

\section{ARQUITECTURA COGNITIVA}

El asistente robótico ideal debe combinar las capacidades humanas y del robot para proporcionarles a los cirujanos el mejor punto de vista del área de operación sin desconcentrarlos durante el procedimiento quirúrgico. Para ello, el asistente robótico inteligente trata de alcanzar las capacidades de los asistentes humanos, que son el resultado de su experiencia y habilidad para aprender. De esta manera, gracias a ambas capacidades se puede conocer el estado actual de la tarea dentro del flujo quirúrgico y cuál es el FA, así como las preferencias particulares de los cirujanos con los que colaboran. Para cubrir estas necesidades se recurre a las arquitecturas cognitivas, que tal y como estableció Langley [13], contienen las memorias a largo y corto plazo para almacenar la información sobre el conocimiento, objetivos y creencias del sistema, representaciones de esos elementos en forma de estructuras mentales y procesos funcionales que operan con estas estructuras y mecanismos de aprendizaje.

La Figura 2 muestra la arquitectura que se propone para facilitar el mejor punto de vista con la cámara durante las tareas quirúrgicas. La memoria a largo plazo contiene el escenario y el conocimiento quirúrgico en la memoria semántica, los comportamientos aprendidos los comportamientos aprendidos de la cámara en la memoria procedural y las experiencias de los usuarios con el tiempo en la memoria episódica. La estimación de los estados quirúrgicos se lleva a cabo en la memoria a corto plazo, utilizando el estado actual del protocolo proporcionado por el sistema perceptual y el conocimiento quirúrgico. Dependiendo del estado actual de la tarea, el generador FA lanza el correspondiente comportamiento de la cámara que cambia el enfoque en el interior de la cavidad abdominal mediante el módulo de movimiento de la cámara. Finalmente, el cirujano interactúa con el 
sistema a través de un interfaz persona-máquina (HMI), que le permite ajustar el punto de vista según sus preferencias.

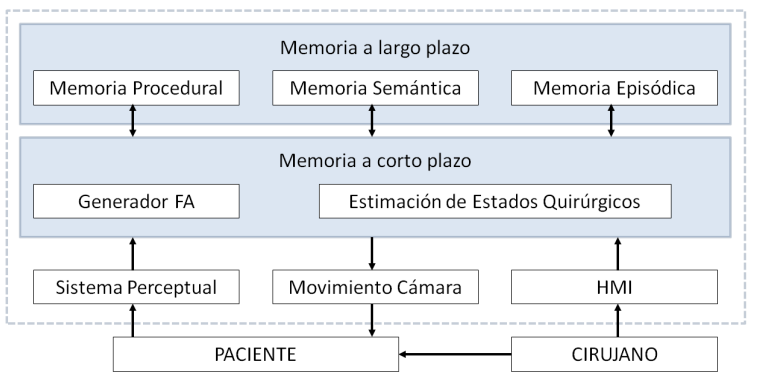

Figura 2: Arquitectura Cognitiva

En las siguientes sub-secciones se describen en detalle cada uno de los módulos de la arquitectura cognitiva.

\subsection{MEMORIA A LARGO PLAZO}

La memoria a largo plazo se divide en tres partes diferenciadas: procedural, semántica y episódica. En primer lugar, la memoria semántica contiene los hechos declarativos que el sistema "conoce", tales como el escenario y el conocimiento quirúrgico, y que son esenciales para razonar y decidir autónomamente. Así, el sistema puede organizar la memoria en diferentes estructuras mentales. Por un lado, el escenario quirúrgico que contiene la información estática de los diferentes objetos del escenario quirúrgico. En el escenario quirúrgico se clasifican los objetos y se establecen las relaciones entre ellos. Cada uno de estos objetos viene definido como una estructura de datos, cuyo primer campo es la clase objeto. En este trabajo se han considerado cuatro clases de objeto con las siguientes definiciones: la cámara $\mathrm{O}_{1}=\left\{\right.$ cámara, ${ }^{\mathrm{n}} \mathrm{T}_{\mathrm{c}}$ \}, el sensor $\mathrm{O}_{2}=$ sensor, $\left.{ }^{0} \mathrm{~T}_{\mathrm{s}}\right\}$, la herramienta quirúrgica $\mathrm{O}_{3}=$ \{herramienta quirúrgica, $\mathrm{m}_{\mathrm{k}}$, $\left.{ }^{\mathrm{m}} \mathrm{P}\right\}$ y la imagen $\mathrm{O}_{4}=$ \{imagen, ${ }^{\mathrm{I}} \mathrm{R}_{\mathrm{c}}, \alpha, \mathrm{a}_{\mathrm{r}}, \mathrm{H}$ \}, siendo: ${ }^{\mathrm{n}} \mathrm{T}_{\mathrm{c}}$ la matriz de transformación entre el efector del robot final del robot externo y la cámara, ${ }^{0} \mathrm{~T}_{\mathrm{s}}$ la matriz de transformación entre la base del robot externo y el sensor $3 \mathrm{D}, \mathrm{m}_{\mathrm{k}}$ indica la herramienta $\mathrm{y}^{\mathrm{m}} \mathrm{P}$ contiene la posición del extremo de la herramienta respecto al sistema de referencia de la marca de la herramienta. En cuanto a la imagen, ${ }^{\mathrm{I}} \mathrm{R}_{\mathrm{c}}$ es la matriz de transformación entre la cámara y la imagen, $\alpha$ es el ángulo de visión, $\mathrm{a}_{\mathrm{r}}$ es la proporción de anchura $w$ y altura $l$ y $\mathrm{H}$ es la distancia entre la lente de la cámara a la escena. En la Figura 3 se muestran los parámetros que se han descrito de la imagen y que permiten obtener la posición de $\{c\}$ con respecto al sistema de referencia de la imagen $\{\mathrm{I}\}$, tal y como se indica a continuación:

$$
{ }^{\mathrm{I}} \mathrm{O}_{\mathrm{c}}=(w / 2 l / 2)
$$

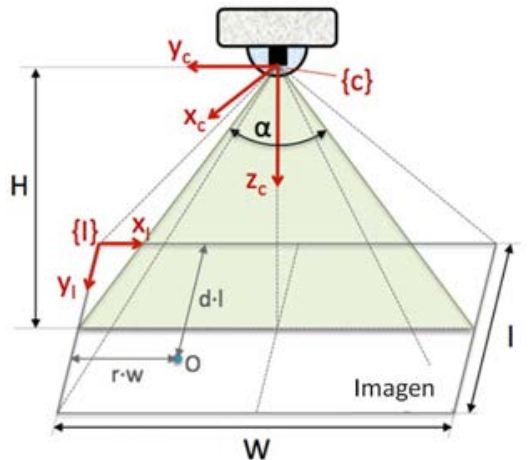

Figura 3: Parámetros de la cámara y la imagen

Por otro lado, el conocimiento quirúrgico que está formado por el modelo de la tarea quirúrgica y la librería de gestos del cirujano son utilizados por el algoritmo de estimación de estados quirúrgicos. De esta forma, la mayoría de las tareas quirúrgicas pueden modelarse con un diagrama de transición de estados, donde la tarea queda dividida en una secuencia de acciones básicas llamadas gestos. En este trabajo se ha considerado la sutura y se ha dividido en seis estados: inicio de la tarea, inserción de la aguja en el tejido con la herramienta derecha mientras la izquierda presiona la zona, extracción de la aguja con la herramienta izquierda mientras se presiona el tejido con la derecha, anudado con la herramienta derecha mientras la izquierda sostiene la aguja, corte con la herramienta derecha que se sustituye con la herramienta derecha y fin de la tarea. La Figura 4 muestra el diagrama de transición de estados, donde la transición $\mathrm{T}_{01}$ se activa con comando de voz y el resto de transiciones se activan con el reconocimiento de gestos del cirujano. Así, cada estado del diagrama coincide con un gesto del cirujano, de manera que cuando un gesto se completa, se compara con los patrones de la librería de gestos y se identifica el gesto realizado [18].

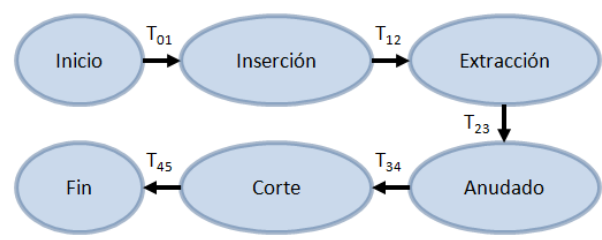

Figura 4: Modelo de la tarea "sutura”

En segundo lugar, la memoria procedural contiene el conocimiento de cómo desarrollar los comportamientos particulares del mini-robot cámara. Estos comportamientos son funciones de las primitivas que cambian el punto de vista de la imagen. Cuando se posiciona el endoscopio durante un procedimiento quirúrgico se tienen en cuenta la posición de los instrumentos y una solución habitual es centrarlos en la imagen. Weede [12] logra posicionar la cámara prediciendo los movimientos de las herramientas, sin embargo, cada cirujano puede manejar los instrumentos de diferentes formas, de 
manera que esta solución produciría errores si el sistema lo maneja alguien que no ha entrenado el sistema. En este trabajo se ha optado por definir una FA para cada estado de la tarea con los siguientes parámetros: el objeto $O$, la posición del objeto en la imagen con las coordenadas $r$ y $d$ (ver Figura 3) y el zoom. Así, no sólo se sigue la navegación de una herramienta quirúrgica sino que también se determina el área de interés de la tarea.

Finalmente, la memoria episódica representa la experiencia de los usuarios con el tiempo y sus perfiles quedan almacenados en una librería que contiene sus preferencias en cuanto al punto de vista de la cámara. Cuando el sistema se inicia, el usuario carga su perfil si existe y si no existe, se crea un perfil con los parámetros FA por defecto. Durante la operación, el usuario interactúa con el sistema mediante comandos de voz: arriba, abajo, izquierda, derecha para cambiar la posición de la cámara y para modificar el zoom se usan zoom in y zoom out. Y una vez que se ejecuta la tarea, los parámetros $r, d$ y zoom para cada gesto se actualizan. De esta manera, cuando el usuario ejecuta la tarea otra vez, el sistema carga su perfil de la librería de usuarios, reduciéndose el número de comando de voz para comunicarse con el sistema.

\subsection{MEMORIA A CORTO PLAZO}

La memoria a corto plazo representa el motor de inferencia del sistema, donde los procedimientos de razonamiento y decisión tienen lugar. Esta memoria está dividida en dos bloques: la estimación de estados quirúrgicos y el generador FA. Por un lado, el generador FA es el encargado de lanzar el correspondiente comportamiento del robot de acuerdo al estado actual de la tarea utilizando la memoria perceptiva. Y por otro lado, el módulo de estimación de estados quirúrgicos evalúa las transiciones entre los diferentes estados gracias al modelo de la tarea (desde el conocimiento quirúrgico) y a un sistema de reconocimiento que interpretan el gesto actual que se está desarrollando el cirujano. Tal y como se describió en el apartado 3.A, el modelo de la tarea consiste en un diagrama de transición de estados, donde las transiciones se activan con la identificación de ciertos gestos específicos del cirujano. El módulo de estimación de estados quirúrgicos recibe información sobre la navegación de las herramientas quirúrgicas mediante un sensor de localización 3D y ofrece el estado actual de la tarea. Para realizar el reconocimiento de los gestos quirúrgicos del cirujano se han utilizado los modelos ocultos de Markov (HMMs), ya que es una técnica estocástica comúnmente utilizada en la evaluación de las destrezas del cirujano [15] y la predicción de estados quirúrgicos [16]-[17]. Como se describe en los trabajos previos [18], cada gesto quirúrgico se asocia con un patrón $\lambda_{\mathrm{k}}$ definido con los siguientes parámetros: $\lambda_{\mathrm{k}}=(\mathrm{S}, \mathrm{E}, \mathrm{A}, \mathrm{B}, \pi)$, donde $\mathrm{S}$ es el número de acciones básicas que caracterizan los gestos, E es el conjunto de características observables que describe cada acción básica, A es la matriz de distribución de probabilidades que relaciona las acciones básicas, A es la matriz de distribución de probabilidades que establece las características observables más probables en cada acción básica, y $\pi$ es la matriz de estados inicial. En este trabajo, las características observables están definidas a partir de la interacción entre las herramientas quirúrgicas con la distancia entre las pinzas, el ángulo que forman las cañas y las velocidades de sus extremos. Los patrones de los gestos $\lambda_{\mathrm{k}}$ se entrenan en un proceso fuera de línea para obtener una librería de gestos. Durante la operación, el sistema de reconocimiento identifica el gesto realizado mediante la comparación de la secuencia adquirida de características observables (E) con los modelos patrón de la librería. Cuando el gesto se reconoce, el estado de transición correspondiente del modelo de la tarea se activa.

El generador FA recibe el estado actual de la tarea y facilita los parámetros que proporcionan el mejor punto de vista para el estado actual a la memoria procedural. De esta forma, el generador FA consiste en una tabla que relaciona cada tarea de un estado con sus correspondientes parámetros FA. En la Tabla 1 aparecen los valores por defecto de los parámetros FA para las cuatro tareas asignadas a los estados de la sutura.

Tabla 1: Generador FA (Parámetros por defecto).

\begin{tabular}{|c|c|c|c|c|}
\hline \multirow{2}{*}{$\begin{array}{c}\text { Estado de } \\
\text { la tarea }\end{array}$} & $\boldsymbol{4}$ & \multicolumn{4}{|c|}{ Parámetros FA } \\
\cline { 2 - 5 } & O & $\boldsymbol{d}$ & zoom \\
\hline Inserción & Herramienta izquierda & 0.4 & 0.6 & 1.25 \\
\hline Extracción & Herramienta derecha & 0.75 & 0.6 & 1 \\
\hline Anudado & Herramienta izquierda & 0.5 & 0.7 & 1.5 \\
\hline Corte & Herramienta izquierda & 0.5 & 0.5 & 1.5 \\
\hline
\end{tabular}

Así, el estado en el que se realiza la punción, el FA se encuentra en la zona derecha de la herramienta izquierda donde la aguja va a insertarse en el tejido y su factor de zoom es de 1.25; para la extracción del hilo, el zoom se restaura y se localiza la herramienta que está presionando el tejido a la derecha de la imagen, para que se visualice la aguja y la pinza izquierda durante esta tarea; para el anudado, la herramienta izquierda que sujeta la aguja se posiciona en el centro y al pie de la imagen, ya que el nudo se realizará en la parte superior; finalmente, para el corte de la hebra, la pinza derecha se sustituye por unas tijeras de corte y la pinza izquierda, que ya está sujetando la aguja, se centra en la imagen. Para estos dos últimos estados, el factor de zoom aplicado es de 1.5. 


\section{EXPERIMENTOS}

\subsection{IMPLEMENTACIÓN}

El escenario quirúrgico de la Figura 5 muestra los elementos necesarios para la asistencia robotizada durante las tareas quirúrgicas, junto con una imagen del mini-robot cámara que muestra el interior de la cavidad abdominal.

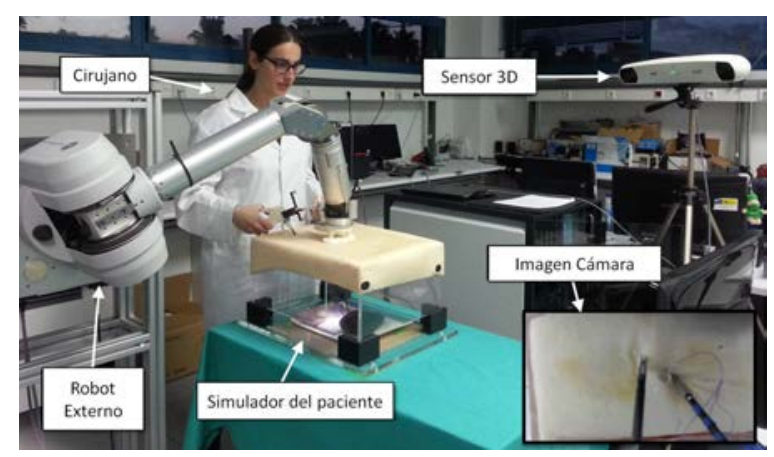

Figura 5: Modelo de la tarea “sutura”

A la derecha del cirujano se encuentra el robot externo (Barrett WAM de 7 grados de libertad de Barrett Technology, Inc.) que sujeta el mini-robot cámara, mientras que el sensor de localización 3D (Polaris Spectra de NDI) se posiciona en el escenario para registrar las marcas pasivas de las herramientas del cirujano. Las características de la cámara son las siguientes: $50^{\circ}$ de ángulo de visión, proporción de anchura y altura de 16:9 y una resolución de $1280 \mathrm{x}$ 720 píxeles. La altura del abdomen del simulador de laparoscopia es de $220 \mathrm{~mm}$. La arquitectura cognitiva ha sido implementada en Matlab 2013 y el visualizador del video se ha integrado utilizando un objeto Timer de Matlab con un período de 0.04 segundos, de tal forma que la imagen se visualiza a 25 fps. Debido a que el algoritmo de estimación de estados quirúrgicos y la adquisición de datos del sensor 3D requieren un alto tiempo computacional, se han ejecutado en un ordenador externo para no comprometer la calidad de la imagen que se muestra. De esta manera, la comunicación entre la arquitectura cognitiva y el ordenador externo se ha llevado a cabo mediante el protocolo UDP (User Data Protocol). Por otro lado, hay que mencionar que el Barrett WAM viene provisto con una librería de control en tiempo real (libbarrett-1.2.1) escrita en C++. La comunicación entre la arquitectura cognitiva y el WAM se ha realizado con el protocolo TCP/IP. La arquitectura envía la salida de la función FA con el movimiento relativo al nodo WAM de ROS que se encarga de recibirlo y mover el efector final.

\subsection{RESULTADOS EXPERIMENTALES}

Para probar la arquitectura cognitiva presentada en la sección 3 se han desarrollado un conjunto de experimentos in-vitro. En estos experimentos, cinco usuarios han ejecutado la tarea de sutura definida en la Figura 2. En primer lugar, los usuarios realizan tres ensayos de la tarea utilizando sólo comandos de voz para obtener la visión óptima del área de trabajo en cada estado. Los datos que se han recogido de los ensayos son el número de comandos de voz utilizados y el tiempo empleado en completar la tarea. Los resultados aparecen en la Tabla 2, donde $\mathrm{T}_{1}, \mathrm{~T}_{2} \mathrm{y} \mathrm{T}_{3}$ son los tiempos de cada ensayo y mean es el valor medio de los tres ensayos. En segundo lugar, los usuarios han realizado la misma tarea pero utilizando la arquitectura cognitiva y los resultados se muestran en la Tabla 3. En cuanto al número de comandos de voz, si se comparan con los resultados obtenidos en la Tabla 2 para el primer ensayo, se observa que la ejecución de la tarea ha requerido menos órdenes con la arquitectura cognitiva. Esto se debe a que el sistema provee autónomamente un punto de vista de la cámara que está predefinido para cada estado. Así, después del primer ensayo, se puede comprobar que el sistema aprende las preferencias de los usuarios mediante la memoria episódica. Como consecuencia, el número de comandos de voz del tercer ensayo se reducen en un $100 \%$, $67 \%$, 60\%, $85 \%$ y $67 \%$ para los usuarios 1 , 2, 3, 4 y 5, respectivamente.

Tabla 2: Resultados experimentales utilizando sólo comandos de voz.

\begin{tabular}{|c|c|c|c|c|c|c|c|c|}
\hline \multirow{2}{*}{ Usuario } & \multicolumn{4}{|c|}{ Comandos de Voz } & \multicolumn{4}{|c|}{ Tiempo (s) } \\
\cline { 2 - 9 } & $\boldsymbol{T}_{\boldsymbol{1}}$ & $\boldsymbol{T}_{\boldsymbol{2}}$ & $\boldsymbol{T}_{\boldsymbol{3}}$ & mean & $\boldsymbol{T}_{\boldsymbol{1}}$ & $\boldsymbol{T}_{\boldsymbol{2}}$ & $\boldsymbol{T}_{\boldsymbol{3}}$ & mean \\
\hline 1 & 16 & 12 & 11 & 13 & 132 & 87 & 87 & 102 \\
\hline 2 & 12 & 10 & 11 & 11 & 123 & 107 & 85 & 105 \\
\hline 3 & 7 & 9 & 8 & 8 & 94 & 90 & 80 & 88 \\
\hline 4 & 14 & 10 & 8 & 11 & 104 & 119 & 103 & 109 \\
\hline 5 & 11 & 9 & 8 & 9 & 127 & 95 & 95 & 106 \\
\hline
\end{tabular}

Con respecto al tiempo, los resultados no indican una relación directa entre el uso de la arquitectura cognitiva y el tiempo para completar la tarea. Mientras algunos usuarios reducen considerablemente su tiempo (usuario 1), otros lo incrementan (usuario 2). Esto se debe principalmente a la complejidad inherente de la tarea, que provoca que el tiempo varíe ampliamente en los diferentes ensayos de un mismo usuario, y también a que los usuarios deben ajustar el punto de vista en el primer ensayo cuando utilizan la arquitectura cognitiva. Sin embargo, los resultados ponen de relieve que con la arquitectura cognitiva reduce el tiempo de ejecución del tercer ensayo con respecto al primer ensayo al tener en cuenta las preferencias de los usuarios. 
Tabla 3: Resultados experimentales utilizando la arquitectura cognitiva.

\begin{tabular}{|c|c|c|c|c|c|c|}
\hline \multirow{2}{*}{ Usuario } & \multicolumn{3}{|c|}{ Comandos de voz } & \multicolumn{3}{|c|}{ Tiempo (s) } \\
\cline { 2 - 7 } & $\boldsymbol{T}_{\boldsymbol{1}}$ & $\boldsymbol{T}_{\boldsymbol{2}}$ & $\boldsymbol{T}_{\boldsymbol{3}}$ & $\boldsymbol{T}_{\boldsymbol{1}}$ & $\boldsymbol{T}_{\boldsymbol{2}}$ & $\boldsymbol{T}_{\boldsymbol{3}}$ \\
\hline 1 & 4 & 1 & 0 & 88 & 87 & 47 \\
\hline 2 & 6 & 4 & 2 & 120 & 114 & 111 \\
\hline 3 & 5 & 4 & 2 & 93 & 87 & 80 \\
\hline 4 & 7 & 4 & 1 & 100 & 96 & 94 \\
\hline 5 & 6 & 2 & 2 & 92 & 91 & 83 \\
\hline
\end{tabular}

\section{CONCLUSIONES}

Este trabajo ha presentado una arquitectura cognitiva para un asistente robótico camarógrafo. El objetivo de la arquitectura cognitiva es proveer del punto de vista más óptimo al usuario en cada fase de la tarea quirúrgica. Para ello, cada estado de la tarea se ha caracterizado con una FA, que está definido con un objeto de interés, la posición de dicho objeto en la imagen y un factor de zoom. De esta manera, cuando un estado de la tarea se reconoce, el sistema ejecuta el comportamiento correspondiente de la cámara para cambiar el enfoque. La arquitectura ha sido validada a través de una batería de experimentos y los resultados muestran que el número de comandos de voz se reducen significativamente en la segunda fase, así como la carga de trabajo del cirujano. Además, el mecanismo de aprendizaje tiene en cuenta las preferencias de cada uno de los usuarios. Hay que mencionar que la arquitectura cognitiva se puede utilizar con otros modelos de tareas quirúrgicas adaptando los parámetros FA por defecto y aumentando el conocimiento del sistema con experiencias de otros usuarios mediante mecanismos de aprendizaje.

\section{Referencias}

[1] R. Taylor, "A perspective on medical robotics," Proceedings of the IEEE, vol. 94, no. 9, pp. 16521664, September 2006.

[2] Intuitive Surgical, Inc. (2007). Company profile of Intuitive Surgical Inc. Sunnyvale, CA, USA, Website, URL http://www.intuitivesurgical.com/

[3] D. Sharma y otros, "Initial experience with the freehand robotic camera holder in laparo- scopic urology,” Journal of Endourology, vol. 23, no. 1, p. A249, 2009.

[4] D. Noonan y otros, "Gaze contingent control for an articulated mechatronic laparoscope," in Proceedings of the IEEE/RAS International Conference on Biomed- ical Robotics and Biomechatronics, pp. 759-764, 2010.

[5] C. Staub y otros, "Human-Computer interfaces for interaction with surgical tools in robotic surgery”, IEEE/EMBS Int. Conf. on Biomedical Robotics and Biomechatronics, Rome, Italy, pp. 81-86, 2012.

[6] V.F. Muñoz y otros, "Control movement scheme based on manipulability concept for surgical robotic assistant”, IEEE Int. Conf. on Robotics and Automation, pp. 245-250, Orlando, Florida, 2006.

[7] S. Haddadin y otros, "Towards the robotic coworker”, Springer Tracts in Advanced Robotics, Vol. 70, pp.261-282, 2011.

[8] M.G. Jacob y otros, "Gestonurse: a multimodal robotic scrub nurse”, ACM/IEEE Int. Conf. on Human-Robot Interaction, Boston, MA, pp. 153154, 2012.

[9] N. Padoy y G.D. Hager, "Human-machine collaborative surgery using learned models", IEEE Int. Conf. on Robotics and Automation, pp. 52855292, Shangai, China, 2011.

[10] E. Bauzano y otros, "Collaborative Human-Robot System for HALS suture procedures", IEEE Systems Journal, Vol. PP (99), pp. 1-10, 2014.

[11] S.Y. Ko y otros, "Intelligent interaction between surgeon and laparoscopic assistant robot system”, IEEE Int. Workshop on Robots and Human Interactive Comunication, pp. 60-65, 2005.

[12] O. Weede y otros, “An intelligent and autonomous endoscopic guidance system for minimally invasive surgery," in Proceedings of the IEEE International Conference on Robotics and Automation, pp. 5762 -5768, 2011.

[13] P. Langley y otros, "Cognitive Architectures: Research Issues and Challenges”. Cognitive Systems Research, Vol. 10 (2), pp.141- 160, 2009.

[14] R. Salgado y otros, "A procedural Long Term Memory for cognitive robotics”, 2012 IEEE Conference on Evolving and Adaptive Intelligent Systems (EAIS), Madrid, pp. 57-62, 2012.

[15] J. Rose y otros, "Generalized Approach for Modeling Minimally Invasive Surgery as a Stochastic Process Using a Discrete Markov Model”, IEEE Trans. Biomedical Engineering, vol. 53, no. 3, pp. 399-413, 2006.

[16] O. Weede y otros, "A cognitive path-guidancesystem for minimally invasive surgery”, IEEE Int. Symposium on Intelligent Systems and Informatics, pp. 139-144, Subotica, Serbia, 2010.

[17] C.E. Reiley y otros, "Motion generation of robotic surgical tasks: learning from expert demonstrations", 32nd Annual Int. Conf. of the IEEE EMBS, pp. 967-970, Buenos Aires, Argentina, 2010.

[18] B. Estebanez y otros, "Manuevers recognition in laparoscopic surgery: artificial neural network and Hidden Markov model approaches”, IEEE RAS/EMBS Int. Conf. on Biomedical Robotics and Biomechatronics, pp. 1164-1169, Rome, Italy, 2012.

[19] M. Quigle y otros, "ROS: an open-source Robot Operating System", International Conference on Robotics and Automation, ser. Open-Source Software workshop, 2009. 